\title{
Resistance to Thyroid Hormone and Cardiovascular Risk
}

\author{
Irene Campi, MD, ${ }^{1}$ Deborah Mannavola, $\mathrm{PhD}^{2}$ and Paolo Beck-Peccoz, $\mathrm{MD}^{3}$ \\ 1. Clinical Fellow; 2. Research Assistant; 3. Full Professor of Endocrinology, and Director, School of Specialization in Endocrinology and Metabolic Diseases,
} Fondazione Policlinico-Istituto di Ricovero e Cura a Carattere Scientifico (IRCCS), University of Milan

DOI: $10.17925 /$ USE.2009.05.1.117

\begin{abstract}
Resistance to thyroid hormone (RTH) is a dominantly inherited syndrome of impaired tissue responsiveness to thyroid hormones (TH) characterized by high circulating TH in the presence of unsuppressed thyroid-stimulating hormone (TSH). TH achieve their action on the heart chiefly via thyroid hormone receptor alpha 1 (TR 1 1), which is the TH receptor (TR) isoform predominantly expressed in such an organ. Data derived from animal models suggest that in RTH the overstimulation of the TRa1 pathway by the high TH levels could explain the cardiovascular abnormalities seen in these animals, although the discordant cardiac gene expression profile between wild-type $(\mathrm{Wt})$ and transgenic mice treated with triiodothyronine $\left(\mathrm{T}_{3}\right)$ imply that the effects of RTH on the heart are complex and not completely explicable by the heightened $\mathrm{T}_{3} / \mathrm{TR} \alpha 1$ signaling. To date, only a few studies have evaluated cardiovascular risk in RTH, with conflicting results, confirming the large variability of the RTH phenotype. In particular, some reports show that several cardiovascular parameters seem to move toward hyperthyroidism, while others show a pattern that resembles thyroid hormone deficiency. Finally, recent data suggest that in addition to reduced vascular compliance and echocardiographic abnormalities, RTH subjects may exhibit some features of metabolic syndrome, suggesting an overall increased cardiometabolic risk in this disorder.
\end{abstract}

\section{Keywords}

Resistance to thyroid hormone (RTH), heart, cardiovascular risk, thyroid hormone receptors, animal model for RTH

Disclosure: The authors have no conflicts of interest to declare.

Received: June 18, 2009 Accepted: October 26, 2009

Correspondence: Paolo Beck-Peccoz, MD, University of Milan, Endocrinology and Diabetology Unit, Pad Granelli, Via F Sforza 35, 20122 Milan, Italy. E: paolo.beckpeccoz@unimi.it

\section{Thyroid Hormones and the Heart}

The heart represents an important target for thyroid hormones (TH). 'Both transcriptional and non-transcriptional effects of $\mathrm{TH}$ may act to modulate the function of the myocardium. In the short term, triiodothyronine $\left(T_{3}\right)$ changes the performance of various sodium, potassium, and calcium channels in the heart, possibly with an inotropic and chronotropic effect. ${ }^{2.3}$ Specific $T_{3}$-response elements (TRE) has been demonstrated in different genes, coding for proteins involved in the regulation of contractility and electrical activity of the heart. ${ }^{4,5} \mathrm{~A}$ positive regulation has been established for myosin heavy chain- $\alpha(\mathrm{MHC} \alpha)^{6,7}, \mathrm{~L}$-type and ryanodine calcium channel, ${ }^{8,9}$ and other ions channels, ${ }^{10}$ such as voltage-gated potassium channels; ${ }^{2}$ while other genes, such as $\mathrm{MHC} \beta$ and $\mathrm{Na}^{+} / \mathrm{Ca}^{2+}$ exchanger, are downregulated by $\mathrm{T}_{3}$ (see Table 1). ${ }^{26,11}$ It has been suggested that $T_{3}$ actions on heart could be, to a certain extent, mediated by increased activity of the sympathoadrenal system, given the fact that cardiac symptoms of hyperthyroidism are relieved by treatment with sympatholytic agents. Due to the fact that thyrotoxicosis plasma and urine levels of catecholamines have been reported as normal or decreased, increased sensitivity of the sympathetic system in hyperthyroidism has been hypothesized. This effect may be mediated by an increased number of $\beta$-adrenergic receptors. Moreover, increased levels of other components of the transmission system could be involved, such as stimulatory guanine nucleotide-regulatory protein. ${ }^{4}$

\section{Resistance to Thyroid Hormone}

Resistance to thyroid hormone (RTH) is a rare (probably one per 50,000 ) dominantly inherited syndrome of impaired tissue responsiveness to $\mathrm{TH}^{12} \mathrm{~A}$ hallmark of this condition is the presence of high circulating $\mathrm{TH}$ in the presence of unsuppressed TSH. In the majority of RTH subjects (about $85 \%$ ), point mutations or small deletions in the thyroid hormone receptor beta (TR $\beta$ ) gene are found. As a result, the mutant TR interferes with the function of normal TRs (dominant negative effect), which explains the dominant mode of inheritance of this syndrome (see Figure 1). However, about $15 \%$ of cases without mutation of the TR $\beta$ gene but with biochemical evidence of the syndrome have been reported. ${ }^{13}$ Even if it has been supposed that a deficiency of a co-factor could be present, to date the exact etiology of the resistance in these cases has not been understood. ${ }^{13}$

The clinical presentation of RTH is highly variable. Classically, these subjects have been divided into two subgroups according to the absence or presence of symptoms of thyrotoxicosis, i.e. generalized thyroid hormone resistance (GRTH) and selective pituitary resistance (PRTH), respectively. Patients with GRTH usually display compensated hypothyroidism, while those with PRTH exhibit variable symptoms of hyperthyroidism. ${ }^{14-16}$ It is now generally accepted that such a distinction is clinically useful, although the TR $\beta$ mutations may be the same in both 
Table 1: Genes Involved in the Regulation of Heart Activities, Presenting $T_{3}$ Response Elements

\begin{tabular}{ll}
$\begin{array}{l}\text { Positively Regulated } \\
\text { Myosin heavy chain- } \alpha\end{array}$ & Negatively Regulated \\
\hline L-type calcium channel & \\
\hline Ryanodine calcium channel & Myosin heavy chain- $\beta$ \\
\hline Voltage-gated potassium channels & Phospholamban \\
\hline Na+/K+-ATPase & Adenylyl cyclase catalytic subunits \\
\hline$\beta$-1-adrenergic receptor & Thyroid hormone receptor alpha 1 \\
\hline Atrial natriuretic hormone & Na $^{+} / \mathrm{Ca}^{+}+$exchanger \\
\hline Voltage-gated potassium channel & \\
\hline Sarcoplasmic reticulum Ca2+-ATPase & \\
\hline ATP = adenosine triphosphate Modified from Klein and Danzi ${ }^{11}$
\end{tabular}

Figure 1: Possible Mechanisms Involved in Dominant Negative Effect-Mutant Thyroid Hormone Receptor Interferes with the Function of Normal Thyroid Hormone Receptor
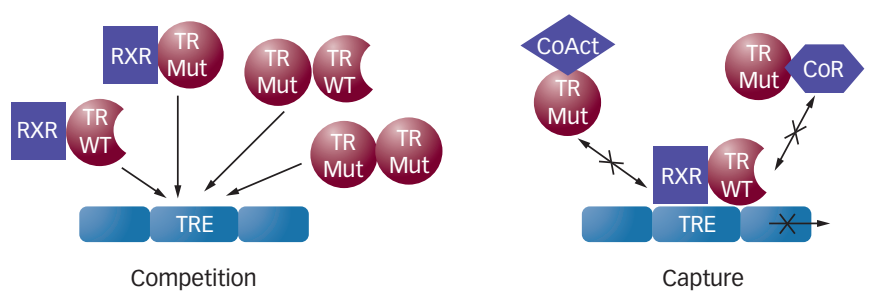

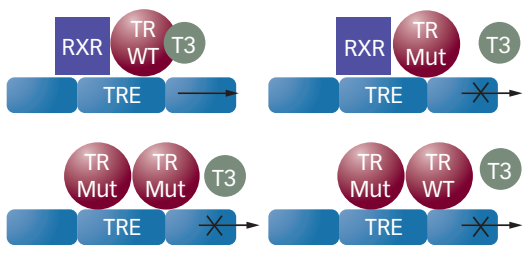

Inhibition

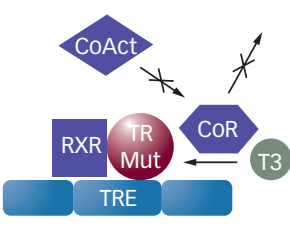

Silencing
$R X R=$ retinoid $X$ receptor; $T R=$ thyroid hormone receptor; $W T=$ wild type; $M U T=$ mutant; $T R E=$ thyroid hormone response element; $C O R=$ co-repressor; $C O A c t=$ co-activator .

GRTH and PRTH patients and even patients belonging to the same family may present with either form. As an example, both GRTH and PRTH patients have resistance in the liver as documented by the measurement of sex-hormone-binding globulin, a peripheral marker of thyroid hormone action, whose levels are in the normal range, thus failing to distinguish between the two forms. ${ }^{17,18}$ The variability of the phenotype of subjects with RTH, including cardiac manifestations, derives from the different expression of TR isoforms in target tissue, which determines the different responsiveness to $\mathrm{TH}^{2}{ }^{12} \mathrm{TR} \beta$ is expressed mainly in the hypothalamus, kidney, liver, anterior pituitary gland, hypothalamus, retina, and cochlea, whereas TR $\alpha$ predominates in the skeletal and cardiac muscle, brain, heart, brown fat, intestine, spleen, and vascular endothelial cells.

\section{Animal Model of Resistance to Thyroid Hormone}

Data from knock-out (KO) mice suggest that TH achieve their action on the heart predominantly via TR $\alpha 1^{4,19-38} \mathrm{In}$ TR $\alpha 1^{-/-}$mice, reduced heart rate (HR) and prolonged QRS- and QT-end-durations have been described. After $\mathrm{T}_{3}$ administration, HR increases in TR $1^{-1-}$ mice, but to a much lesser degree compared with wt mice, even after prolonged exposure to high doses..$^{22}$ Conversely, TR $\beta^{-1-}$ mice display a reduced QT end time ${ }^{23}$ and an increased heart rate. Compared with wt mice, $\mathrm{TR}^{-/}{ }^{-}$showed similar decreases and increases in $\mathrm{HR}$ in response to $\mathrm{TH}$ deprivation and $\mathrm{L}-\mathrm{T}_{3}$ treatment, respectively. ${ }^{24}$ Deletion of both $T R \alpha 1$ and TR $\beta$ results in a similar phenotype to TR $\alpha 1 \%$ mice, suggesting a crucial role of TR $\alpha 1$ in specific heart functions, namely regulation of pacemaker function and ventricular repolarization. ${ }^{22,23}$ Transgenic KO of both TR $\alpha 1$ and TR $\alpha 2$ causes bradycardia and decreased myocardial contractility. ${ }^{25}$ In these particular transgenic mice, HR increases with the administration of $\mathrm{L}_{-} \mathrm{T}_{3}$. This observation suggests that in the absence of TR $\alpha$ the heart rate is in the hypothyroid range, but can still be increased by $\mathrm{TH}$, probably acting through the TR $\beta .^{25}$ A reduction of $\mathrm{HCN} 2$ and $\mathrm{HCN} 4$ expression (genes coding for the cardiac pacemaker current) ${ }^{26,27}$ has been described. Moreover, these mice exhibit a reduced cardiac sensitivity to dynamic testing, supporting the hypothesis that TR $\alpha$ is the major functional cardiac TR isoform. 21,25 The increased levels of HCN2 and HCN4 in TR $\beta^{-/-}$mice may be linked to TR $\alpha$-mediated $T_{3}$ responsiveness, in fact reducing the high levels of $\mathrm{TH}$ in $\mathrm{TR} \beta^{-/-;}$; heart rate and $\mathrm{HCN}$ gene expression tend to normalize. ${ }^{27}$

Mice 'knock-in' models ${ }^{28-35}$ have been generated in order to better understand the cardiac phenotype of RTH subjects. The PV mutation consists of a C-insertion at codon 448 in exon 10, which produces a frameshift of the carboxyl-terminal 14 amino acids of TR $\beta 1$ resulting in a complete loss of TH-binding activity. ${ }^{31}$ The TR $\alpha^{\mathrm{PV} /+}$ and TR $\beta^{\mathrm{PV} / \mathrm{PV}}$ mutations lead, respectively, to opposite effects on energy metabolism in the heart: ${ }^{28}$ cardiac glucose utilization was reduced in the TR $\alpha^{\mathrm{PV} /+}$ mutants and markedly enhanced in TR $\beta^{\mathrm{PV} / \mathrm{PV}}$. Moreover, $\mathrm{TR} \alpha^{\mathrm{PV} /+}$ show a reduction in heart size and blood pressure compared with wt mice, while heart size and blood pressure were normal in TR $\beta^{\mathrm{PV} / \mathrm{PV}}$ mice. These data appear to be consistent with the alterations in cardiac function, such as bradycardia and reduced cardiac output, found in hypothyroidism and tachycardia associated with hyperthyroidism and RTH.

Svanson and collaborators ${ }^{32}$ reported a decreased heart rate and a diminished contractile function in TR $\beta^{P V / P V}$ mice when thyroid hormone levels were lowered into the normal range, suggesting that the homozygous PV mutant can negatively interfere with TR 1 signaling in the heart. These effects on the heart rate may be explained in part by a decreased expression of the ion channels. By contrast, the absence of functional consequences in TR $\beta^{P V / P V}$ mutant mice with elevated thyroid hormone levels is likely due to an increased occupancy of TR $\alpha 1$. The expression of a mutant $\operatorname{TR} \beta(\Delta 337 \mathrm{~T})$ in heart mice, driven by a $\beta$-actin promoter $^{33}$ or an $\mathrm{MHC} \alpha$ promoter, ${ }^{34}$ induces a hypothyroid phenotype in the heart, despite normal $T_{3}$, thyroxine $\left(T_{4}\right)$, and TSH levels in these mice. In isolated hearts particularly, the impairment of thyroid hormone action leads to left ventricular dysfunction; indeed, the cardiac performance measured in vivo is similar between wt and mutant animals, suggesting that other mechanisms could compensate in vivo the abnormalities of the mutant transgenic heart. ${ }^{34}$ These findings are consistent with a dominant negative interference of wt TR function by the mutant TR and provide insight into the observation of the relative impairment in cardiac contractility seen in many patients with RTH. ${ }^{39,40}$ Indeed, the effects of RTH on the heart are complex and not completely explained by the heightened $T_{3} / T R \alpha 1$ signaling, as suggested by the discordant cardiac gene expression profile between WT mice treated with $T_{3}$ and TR $\beta^{P V / P V} .{ }^{35}$ Recently, it has been suggested that while TR $\alpha$ may be crucial for contractility, the $\beta$ isoform could be involved in the 
regulation of cardiac energy substrate metabolism. ${ }^{36} \mathrm{TR}^{-1-}$ mice display a glucose/fatty acid oxidation balance comparable to wt. By contrast, $\operatorname{TR} \beta \mathrm{KO}$ exhibits a shift of metabolism toward glucose oxidation at the expense of fatty-acid oxidation. In a pressure-overload-induced cardiac hypertrophy model, the current authors found an increase of glucose oxidation. In these mice, TR $\beta$ expression in the heart is reduced by more than 70\% compared with normal animals. Gene therapy with adenovirus (AAV)-expressing TR $\beta$ tends to normalize the myocardial metabolism. Interestingly, in this model hypertrophy is associated with a decreased contractile phenotype and an altered intracellular calcium transient, ${ }^{37}$ mainly due to a change in $\mathrm{MHC}$ isoform expression (from $\mathrm{MHC} \alpha$ to $\mathrm{MHC} \beta$ ) and a reduction in sarcoplasmic reticulum $\mathrm{Ca}^{2+}$-adenosine triphosphate (ATPase) (SERCA), respectively. Treatment with AAVexpressing thyroid hormone receptor TR $\alpha 1$ or TR $\beta 1$ improves contractile function in hypertrophied hearts and increases SERCA levels, suggesting that receptor expression level, rather than isoforms, affects contractile function in cardiac hypertrophy. ${ }^{37}$

Finally, TR $\beta$ KO mice and TR $\alpha / T R \beta$-double KO mice exhibited a significant decrease in capillary density compared with wt mice, whereas there was no change in capillary density in $\mathrm{TR} \alpha \mathrm{KO}$ mice. These data indicate that TR $\beta$ expression in coronary endothelial cells plays an essential role in angiogenesis during cardiac development. ${ }^{38}$

\section{Resistance to Thyroid Hormone and Cardiovascular Risk}

The potential for cardiovascular derangements in RTH is complex, and to date only a few studies have evaluated the cardiovascular risk in these subjects. ${ }^{12,39,40,42,44}$ it is well-known that either hyperthyroidism or hypothyroidism causes alterations in the cardiovascular system and metabolism. ${ }^{2,3}$ In overt and subclinical hypothyroidism, the increased serum levels of lipids and homocysteine together with diastolic hypertension and the increased systemic vascular resistance are potentially associated with an increased risk of atherosclerosis. Atrial fibrillation (AF) is a recognized complication of overt hyperthyroidism, and subclinical hyperthyroidism is also known to be a risk factor for development of AF. Supraventricular dysrhythmias, particularly AF, in older hyperthyroid patients may account for some of the excess cardiovascular and cerebrovascular mortality, especially due to embolic phenomena.

Data concerning the influence of RTH on the heart anatomy, investigated chiefly by echocardiography, ${ }_{1}^{13,39,40}$ are conflicting and confirm the extreme variability and heterogeneity of clinical manifestations in RTH syndrome. Similar to observations in hyperthyroid patients, Brucker-Davis et al. ${ }^{35}$ discovered increased systolic cardiac performance in adults affected with $\mathrm{RTH}$. This finding suggests that the heart is relatively less resistant than other organs. Thus, the increase of contractility was less than expected given the level of thyroid hormone, implying the presence of some degree of resistance in the ventricle. Moreover, given the fact that the increase of systolic cardiac performance was detected in adults only, the authors hypothesized that children with RTH could be more 'resistant' to thyroid hormone. A decrease in the mutant/normal receptor ratio with age was assumed an explanation of these results. In contrast to previous reports, in the National Institutes of Health $(\mathrm{NIH})$ study the resting pulse was found to be faster in RTH patients as a group, but not after adjustment for age. compared with normal controls, a higher frequency of valvular defects was observed, namely mitral valve prolapse in RTH subjects. More recently, Kahaly and collaborators ${ }^{12}$ compared 54 RTH patients divided into three groups: healthy, hypothyroid, and hyperthyroid patients. In agreement with previous data, they observed that in RTH some parameters (cardiac output, stroke volume, and isovolumic relaxation and deceleration times) were moving toward hyperthyroidism, but others were not. Overall differences in terms of cardiovascular changes were smaller between the RTH and control group than between the RTH and the hyperthyroid or hypothyroid patients, suggesting an incomplete response of the heart to $\mathrm{TH}$. Cardiovascular symptoms and signs were less frequent and severe in RTH compared with hyperthyroid subjects. RTH subjects showed a systolic cardiac performance within the normal range; in particular, Doppler velocities, heart rate, stroke volume, and cardiac output were significantly lower with respect to hyperthyroid subjects. In comparison with controls, RTH patients exhibit an enhanced left ventricular (LV) contractile function and shorter diastolic parameters, while systolic and diastolic diameters of the LV were not significantly different. Interestingly, in RTH subjects without cardiac signs, median age, heart rate, and TH levels were higher and diastolic relaxation time was shorter compared with those with palpitations and tachycardia. Nevertheless, all other cardiovascular parameters showed no significant differences. Even if the heart rate was high, especially in subjects harboring the mutations Y321S, M334R, and C446R, no significant correlation was found between cardiovascular features and the type of mutation.

To a certain extent, some of these results were in contrast to the previous $\mathrm{NIH}$ paper. ${ }^{39}$ Notably, in the Kahaly et al. paper a higher heart rate in RTH was discovered. Moreover, the frequency of mitral valve prolapse was not increased in RTH compared with controls. Remarkably, a significantly higher heart rate and a larger diameter of the left atrium (LA) and lower LV ejection fraction were found in affected children compared with controls of comparable age, while in the Brucker-Davis paper adults but not children exhibited improved of systolic cardiac performance. We have recently published data that are apparently different from those of Kahaly and collaborators, although the different results could be explained by the different selection criteria of the RTH patients. ${ }^{40}$ Our 16 RTH patients had no thyrotoxic cardiovascular signs and symptoms, were untreated with thyroid or cardiovascular drugs, and showed comparable heart rates to the control group. They also displayed higher systolic, diastolic, and mean arterial pressure than controls, but similar pulse pressure. The global LV performance, as expressed by the stroke index, did not differ between the two groups. However, the level of global LV systolic function was lower in RTH patients than in controls. Moreover, myocardial relaxation was deranged, similar to that observed in patients with overt and subclinical thyroid hormone deficiency. Furthermore, the mean interventricular septum diastolic thickness and mean LV posterior wall diastolic thickness were significantly lower in RTH patients. Consequently, the mean LV mass was reduced in $\mathrm{RTH}$ compared with unaffected individuals, suggesting the presence of reduced TH effects during heart development. Interestingly, data from animal models could explain, to some extent, our observation, 19,41 as mutant TR $\beta$ prevented TH-induced cardiac hypertrophy, probably by interfering with the function of normal TR and inducing hypothyroidism in the heart of the transgenic mice.

Finally, even if the pulse-pressure-to-stroke index ratio (a parameter of total arterial stiffness) was similar to controls, the systemic vascular 


\section{Thyroid Disorders}

resistance index was higher in our RTH patients, according to recent data from owen et al. ${ }^{42}$ These authors found an increased arterial stiffness in RTH compared with sex- and age-matched normal controls. This vascular pattern is more similar to that observed in hypothyroid than hyperthyroid subjects, in which a lower arterial stiffness has been reported. In addition, the TR $\beta$ knock-in mouse model of RTH displays an impaired endothelial vascular function and hypercholesterolemia compared with wt controls. ${ }^{43}$ In the vascular endothelial cell the predominant TR isoform is TR $\alpha$. Equally, these data suggest that TR $\beta$ may have a certain role in endothelial function. However, a number of the rapid effects of thyroid hormone on the vascular wall are mediated via a non-genomic mechanism. Furthermore, the elevated arterial stiffness may lead to increased cardiovascular risk in RTH.

Ciulla and co-workers ${ }^{44}$ found that the echoreflectivity (dCVF\%) of interventricular septum was slightly higher in the RTH patients compared with controls and hyperthyroid subjects, but lower than in hypothyroid patients. The echoreflectivity is a marker of fibrous myocardial remodeling related to increased myocardial collagen deposition. This parameter represents a risk factor for the performance of coronary circulation, systodiastolic function, and electric stability of the heart. As suggested by animal models of hypothyroidism, ${ }^{45,46}$ the lack of TH induces a significant increase in myocardial echoreflectivity. The authors speculate that while myocytes mainly express the TR $\alpha$ isoform, which could explain the hyperkinetic pattern seen in some of RTH, other non-myocyte cells may present a prevalence of TR $\beta$ with a phenotype that mimics a situation of mild hypothyroidism. In terms of the lipid profile, Owen observed significantly elevated low-density lipoprotein (LDL) cholesterol levels in RTH subjects compared with controls. ${ }^{42}$ Furthermore, dyslipidemia is associated with reduced nitric oxide bioavailability and impairment of smooth-muscle relaxation that could also increase arterial stiffness.

Mitchell and co-workers (personal communication) observed that the insulin sensitivity index (ISI) and homeostasis model assessment-insulin resistance (HOMA-IR) was strikingly elevated in a group of RTH subjects compared with controls. Moreover, plasma free fatty acid (FFA) concentrations were $\sim 30 \%$, high-density lipoprotein (HDL) cholesterol was significantly reduced, and triglycerides were slightly elevated. This finding could be consistent with increased sympathetic activity and adipose tissue lipolisys. Remarkably, the strong correlation between fasting plasma FFA concentrations and insulin resistance is well-known. The mechanism for this is unclear: one hypothesis is that skeletal muscle metabolism of fatty acid is impaired, leading to muscle lipotoxicity and impaired insulin action; alternatively, RTH subjects exhibit hepatic RTH action that may also be associated with reduced hepatic sensitivity to insulin action.

\section{Conclusion}

In addition to reduced vascular compliance and echocardiographic abnormalities, RTH subjects exhibit some features of metabolic syndrome, such as insulin resistance together with elevated circulating triglycerides and reduced HDL cholesterol levels, which suggests that overall cardiometabolic risk in this disorder may be increased.

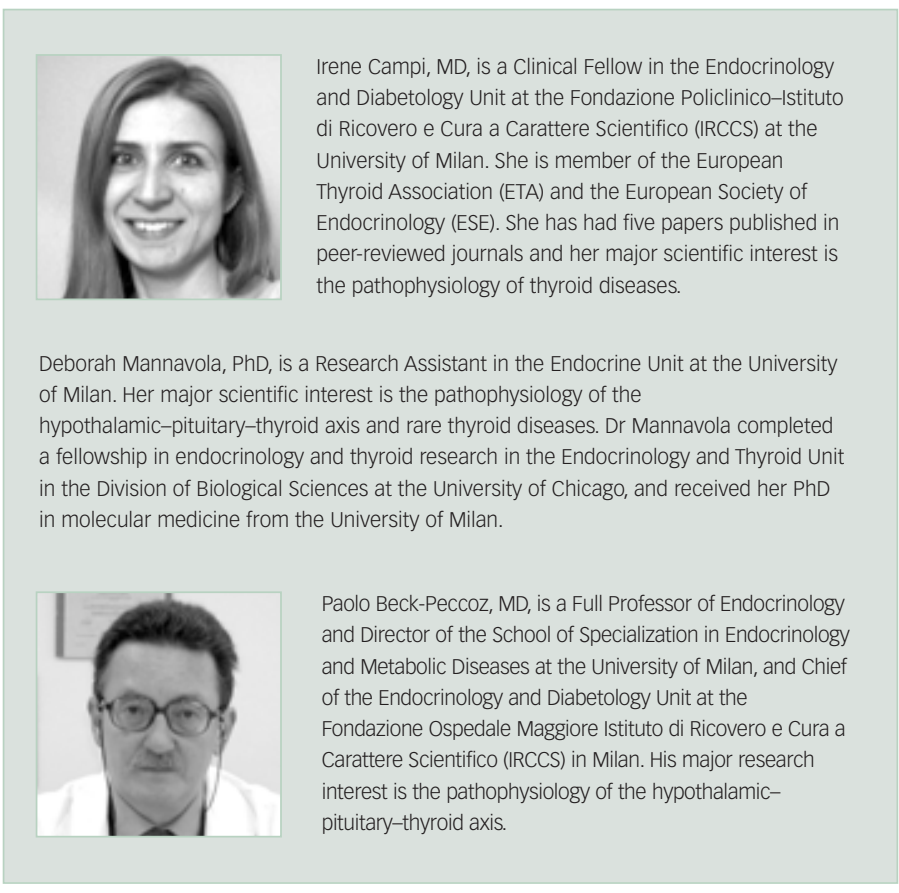

1. Kinugawa K, et al., Circ Res, 2001;89:591-8.

2. Klein I, et al., N Eng/ J Med, 2001;344(7):501-9.

3. Dillmann WH, Thyroid, 2002;12,(6):447-52.

4. Kahaly GJ, et al., Endocr Rev, 2005;26(5):704-28.

5. Danzi S, et al., Thyroid, 2002;12(6): 467-72.

6. Ojamaa K, et al., Endocrinology, 1993;132:1002-6.

7. Ojamaa K, et al., Endocrinology, 1996; 137:802-8.

8. Kreuzberg U, et al., Am J Physiol Heart Circ Physiol, 2000;278:H723-H730.

9. Jiang $\mathrm{M}, \mathrm{Xu} \mathrm{A}, \mathrm{Am} J$ Physiol Heart Circ Physiol, 2000;278:H1429-H1438.

10. Pachucki J, et al., Circ Res, 1999;85:498-503.

11. Klein I, et al., Circulation, 2007;116:1725-35.

12. Kahaly GG, et al., JCEM, 2002; 87(1):204-12.

13. Pohlenz J, et al., J Clin Endocrinol Metab, 1999;84:3919-28.

14. Refetoff $S$, Werner and Ingbars's The Thyroid: A Fundamental and Clinical Text (ninth edition), Philadelphia, PA: Lippincott, Williams and Wilkins, 2005;1109-29.
15. Chatterjee VK, et al., Oxford Textbook of Endocrinology, London: Oxford University Press, 2002;339-49.

16. Beck-Peccoz P, et al., Thyroid, 1994;4(2):225-32.

17. Beck-Peccoz P, et al., J Clin Endocrinol Metab, 1990;71:19-25.

18. Tolulope O, et al., Ann Clin Biochem, 2006;43:431-40.

19. Ortiga-Carvalho TM, et al., Endocrinology, 2004;145(4):1625-33

20. Zhang XY, et al., Mol Endocrinol, 2002;16:2077-92.

21. O'Shea PJ, et al., J Clin Endocrinol, 2002;175:553-70.

22. Wikström L, et al.,EMBO J, 1998;17(2):455-61.

23. Johansson C, et al., Am J Physiol, 1999;276:H 2006-12.

24. Weiss RE, et al., Endocrinology, 1998;139: 4945-52.

25. Macchia PE, et al., Proc Natl Acad Sci U S A, 2001;98:349-54.

26. Ludwig A, et al., EMBO J, 1999;18(9):2323-9.

27. Gloss B, et al., Endocrinology, 2001;142(2):544-50.

28. Esaki T, et al., Am J Physiol Endocrinol Metab, 2004;287:E1149-E1153.

29. Kaneshige M, et al., Proc Natl Acad Sci U S A, 2000;97:13209-14. 30. Kaneshige M, et al., Proc Natl Acad Sci U S A, 2001;98:15095-100.
31. Parrilla RA, et al., J Clin Invest, 1991;88:2123-30.

32. Swanson EA, et al., Endocrinology, 2003;144:4820-25.

33. Gloss B, et al., Endocrinology, 1999;140:897-902.

34. Pazos-Moura C, et al., Circ Res, 2000;86; 700-706.

35. Miller LD, et al., Genome Biol, 2004;5(5):R31.

36. Belke DD, 8th International Workshop on RTH, in abstract book OP\#8.

37. Belke DD, et al., Endocrinology, 2007;148(6):2870-77.

38. Makino A, et al., Endocrinology, 2009;150:2008-15.

39. Brucker-Davis F, et al., Ann Intern Med, 1995;123(8):572-83.

40. Pulcrano M, et al., J Clin Endocrinol Metab, 2009;94(8):2812-16.

41. Weiss RE, et al., Am J Physiol Endocrinol Metab, 2002;283:E428-35.

42. Owen PJ, et al., Clin Endocrinol, 2009;70(4):650-54.

43. Owen PJ, et al., Eur J Clin Invest, 2007;37:390-95.

44. Ciulla MM, et al., Thyroid, 2001;11(7):613-19.

45. Yao J, et al., Cardiovasc Res, 1992;26:603-7.

46. Grover-McKay M, et al., Invest Radiol, 1984;26:227-32. 